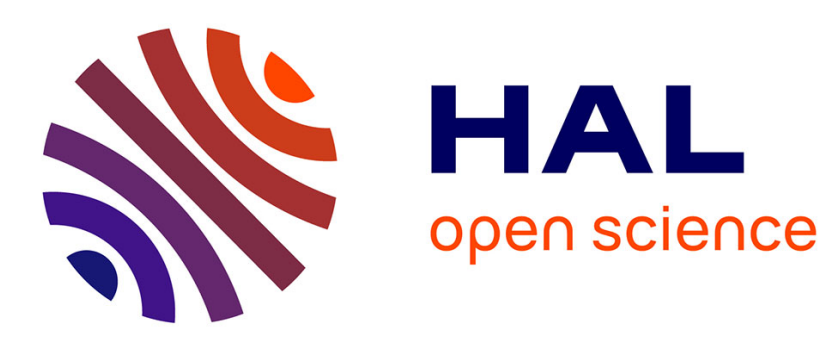

\title{
La légitimité de la culture scolaire mise à l'épreuve. L'exemple de l'éducation physique et sportive dans le second degré en France
}

Gilles Combaz, Olivier Hoibian

\section{- To cite this version:}

Gilles Combaz, Olivier Hoibian. La légitimité de la culture scolaire mise à l'épreuve. L'exemple de l'éducation physique et sportive dans le second degré en France. Education et Sociétés: Revue internationale de sociologie de l'éducation, 2009, 23. hal-03125010

\section{HAL Id: hal-03125010 \\ https://hal-univ-tlse2.archives-ouvertes.fr/hal-03125010}

Submitted on 2 Feb 2021

HAL is a multi-disciplinary open access archive for the deposit and dissemination of scientific research documents, whether they are published or not. The documents may come from teaching and research institutions in France or abroad, or from public or private research centers.
L'archive ouverte pluridisciplinaire HAL, est destinée au dépôt et à la diffusion de documents scientifiques de niveau recherche, publiés ou non, émanant des établissements d'enseignement et de recherche français ou étrangers, des laboratoires publics ou privés. 


\title{
LA LÉGITIMITÉ DE LA CULTURE SCOLAIRE MISE À L'ÉPREUVE. L'EXEMPLE DE L'ÉDUCATION PHYSIQUE ET SPORTIVE DANS LE SECOND DEGRÉ EN FRANCE
}

\author{
Gilles Combaz, Olivier Hoibian
}

De Boeck Supérieur | Éducation et sociétés 》

2009/1 n 23 | pages 189 à 206

ISSN 1373-847X

ISBN 9782804102678

Article disponible en ligne à l'adresse :

https://www.cairn.info/revue-education-et-societes-2009-1-page-189.htm

Distribution électronique Cairn.info pour De Boeck Supérieur.

(C) De Boeck Supérieur. Tous droits réservés pour tous pays.

La reproduction ou représentation de cet article, notamment par photocopie, n'est autorisée que dans les limites des conditions générales d'utilisation du site ou, le cas échéant, des conditions générales de la licence souscrite par votre établissement. Toute autre reproduction ou représentation, en tout ou partie, sous quelque forme et de quelque manière que ce soit, est interdite sauf accord préalable et écrit de l'éditeur, en dehors des cas prévus par la législation en vigueur en France. Il est précisé que son stockage dans une base de données est également interdit. 


\section{La légitimité de la culture scolaire} mise à l'épreuve. L'exemple de l'éducation physique et sportive dans le second degré en France

GILLES COMBAZ

UMR Éducation \& Politiques (Université Lumière Lyon 2 -INRP)

Université Lumière Lyon 2

86 rue Pasteur

69366 Lyon cedex 07

gilles.combaz@univ-lyon2.fr

OLIVIER HOIBIAN

Sports, organisations, identités (EA3690)

UFRSTAPS- Université Paul Sabatier-Toulouse III

I 18, route de Narbonne

31062 Toulouse

olivier.hoibian@wanadoo.fr

L

a manière dont la légitimité de la culture scolaire s'élabore est étroitement liée au processus de sélection, de hiérarchisation et de justification des éléments culturels choisis pour faire l'objet d'une transmission à l'école. Comme l'écrit Forquin (1996, 15), "à l'intérieur même de ce qui est tenu pour légitime au sein de la culture, c'est-à-dire dans la culture considérée comme patrimoine intellectuel et spirituel méritant d'être préservé et transmis, il est de fait (...) que l'éducation scolaire ne réussit jamais à incorporer dans ses programmes et ses cursus qu'un spectre étroit de savoirs, de compétences, de formes d'expression, de mythes et de symboles socialement mobilisateurs". Dès lors se pose la question centrale qui constitue le véritable fil directeur de notre travail : "Qu'est-ce qui, dans les contenus vivants de la culture, dans les significations qui ont pouvoir actuellement d'interpeller nos pensées et de régler nos existences, peut être considéré comme ayant une 'valeur éducative' ou une pertinence sociale suffisantes pour justifier les dépenses de toutes natures que suppose un enseignement systématique et soutenu par l'État ?" (Forquin 1996, 15).

Compte tenu des enjeux, sociaux et politiques, les choix réalisés en termes de culture ne font pas nécessairement l'objet d'un large consensus. La construc- 
tion des savoirs scolaires résulte, pour une part, d'interactions entre divers acteurs sociaux qui peuvent aussi bien coopérer que s'affronter pour défendre telle ou telle conception des savoirs qu'il est légitime de transmettre à l'école. L'élaboration de la culture scolaire et de son bien-fondé fait l'objet de diverses interprétations et de nombreuses propositions émanant de différents acteurs : responsables de l'administration scolaire, experts en didactique de la discipline, enseignants mais aussi élèves, parents d'élèves, syndicats enseignants, etc., qui n'ont pas nécessairement des intérêts et des conceptions proches, loin s'en faut.

En prenant l'exemple de l'enseignement de l'éducation physique et sportive (EPS), objet de récents programmes pour les lycées et les lycées professionnels, nous soulignerons l'existence de tensions résultant de la délimitation et de la définition de la culture corporelle à partir de laquelle s'élaborent les curricula. Pour mener l'analyse, deux principales dimensions sont prises en considération : la nature des activités physiques retenues et les modalités de pratique valorisées.

À la fois par ses caractéristiques intrinsèques et les pratiques qu'elle autorise, chaque discipline sportive conduit à des usages sociaux du corps bien déterminés. Ainsi, l'haltérophilie, la course de demi-fond, la gymnastique artistique ou les activités physiques de pleine nature (escalade, canoë-kayak, vol libre, etc.) ne sont pas tout à fait comparables du point de vue du rapport au corps. Comme l'a très bien montré Pociello (1981), les qualités requises et valorisées par leur pratique renvoient non seulement à des investissements corporels socialement différenciés mais aussi à des significations qui, culturellement, ne peuvent être appréhendées comme équivalentes.

Par ailleurs, une même activité physique peut donner lieu à des modalités de pratique assez diversifiées. Les orientations peuvent être : compétitives, hygiéniques, hédoniques, etc. Le spectre des activités physiques et des modalités de pratique existantes est donc très large. Et les choix opérés pour déterminer des contenus corporels dignes d'être transmis par l'école ne sont pas neutres socialement. Les travaux de Boltanski (1971) montrent en effet que les usages sociaux du corps sont associés à des modes de vie et à des valeurs pouvant être culturellement très distinctifs.

Enfin, un aspect, plus périphérique, mérite d'être pris en compte : l'évolution des pratiques physiques extrascolaires. Celle-ci contribue à façonner les aspirations des élèves en matière de pratiques corporelles.

Parmi l'ensemble des possibilités existantes, quels choix opère-t-on pour élaborer les contenus d'enseignement en EPS ? Sur quoi la légitimité de la culture corporelle ainsi délimitée repose-t-elle?

En termes d'apports culturels, toutes les pratiques physiques sont-elles réellement comparables? Considère-t-on que certaines sont dotées de qualités intrinsèques constituant sans conteste la forme la plus légitime de la culture corporelle à partir de laquelle les contenus d'enseignement doivent être élaborés ? 
Peut-on supposer que les transformations motrices que la pratique de ces activités induit, le type de rapport au corps valorisé à cette occasion, les significations sociales pouvant être associées à ces activités, représentent des apports irremplaçables contribuant à établir l'universalité des contenus enseignés en EPS ? Existe-t-il des modalités de pratique représentant ce qu'il y a de plus élaboré dans le registre de la motricité ? De ce point de vue, l'affrontement compétitif codifié et la recherche de performance constituent-ils des modalités de pratique permettant, plus que d'autres, de tendre vers une certaine forme d'excellence corporelle ? En quoi les choix réalisés contribuent-ils à l'élaboration d'une culture corporelle commune?

Ces questions renvoient à des enjeux qui concernent les responsables de la construction des programmes, les professeurs censés les mettre en œuvre concrètement et les élèves auxquels s'adressent ces enseignements. Tous ces acteurs n'ont pas nécessairement les mêmes intérêts à défendre et des tensions risquent de déstabiliser ce qui fonde la légitimité culturelle des contenus enseignés.

\section{Points d'appui pour la recherche}

T es interrogations formulées plus haut, à propos de l'EPS, font écho, pour une part, aux travaux de Vulliamy centrés sur l'enseignement de la musique en Grande-Bretagne. Ce sociologue remet vigoureusement en cause la légitimité de la culture musicale sur laquelle repose cet enseignement. Il dénonce sans détour la place excessive accordée à la musique classique européenne et regrette que la musique populaire moderne soit aussi peu considérée à l'école (cette dernière étant beaucoup plus proche des intérêts de la plupart des élèves). Ne craignant nullement la confusion entre l'engagement idéologique et la sphère scientifique, il défend ardemment les musiques de tradition afro-américaine qui devraient, selon lui, occuper une place plus importante dans l'enseignement dans la mesure où elles représentent un instrument de libération culturelle et de subversion politique. Certes, les positions soutenues par Vulliamy se révèlent très radicales et sont, à divers égards, fort critiquables. Mais elles posent bien la question relative au processus de hiérarchisation et de sélection des éléments de culture musicale transmis dans le cadre scolaire.

Sans doute y a-t-il lieu d'établir quelques rapprochements avec ce qu'on observe dans le cadre de l'enseignement de l'EPS en France. Peut-on, par exemple, envisager que certaines activités physiques, ayant une dimension internationale par le type de confrontation auxquelles elles donnent lieu (Jeux Olympiques, championnats du monde, etc.), puissent avoir le statut qu'occupe la musique classique savante dans l'étude Vulliamy ? Sous prétexte de coller à leurs aspirations, faut-il absolument abonder dans le sens des élèves en leur proposant, en EPS, des 
activités qu'ils pratiquent avec passion en dehors de l'école ? Si c'est le cas, doiton mettre sur le même plan, l'athlétisme, la natation, la gymnastique aux agrès et le hip-hop, le skateboard ou le football pratiqué au pied des immeubles ?

Les questions soulevées en introduction renvoient également au rapport que les enseignants entretiennent aux différentes formes de culture existantes. Certes, le curriculum prescrit fournit généralement un ensemble d'orientations assez claires quant à la nature des contenus enseignés. Il constitue cependant une trame suffisamment lâche pour permettre aux professeurs d'adapter leurs enseignements aux réalités locales. Cette relative latitude favorise également l'expression des propres conceptions culturelles des enseignants. Plusieurs recherches sociologiques centrées sur l'enseignement du français le montrent de manière tout à fait explicite.

La typologie élaborée par Demailly (1985) révèle ainsi les profondes différences de conceptions distinguant les professeurs de collège étudiés. La définition de ce qu'est la littérature, la nature des œuvres sur lesquelles ils souhaitent porter l'accent avec leurs élèves, leur rapport aux normes linguistiques constituent autant d'éléments distinctifs.

Dans le même registre, la recherche menée par Isambert-Jamati \& Grospiron (1984) souligne la divergence des conceptions et des pratiques des professeurs de français de lycée. Des enseignants les plus "classiques" très attachés aux grandes œuvres littéraires et à un usage policé de la langue française jusqu'aux professeurs "modernistes" préoccupés avant tout de méthodes d'apprentissage et soucieux des progrès réalisés par les élèves de milieux modestes, les profils diffèrent assez nettement.

Centré sur l'apprentissage de la narration écrite au collège, le travail de Tupin (1996) met également en lumière de tels écarts de conceptions et de pratiques. Ici, de profondes divergences se manifestent, entre autres, par la place accordée (ou non) à la culture première des élèves (notamment celle constituée par les fictions télévisuelles massivement suivies par les adolescents). L'auteur montre que les enseignants les plus "démocratisants" sont ceux qui sont capables de jouer le rôle de véritables passeurs entre les univers culturels des élèves et la culture normée de l'école.

Dans les trois recherches évoquées, les différences observées entre les conceptions et les pratiques des enseignants tiennent autant à leurs habitus culturels respectifs qu'à leur capacité à prendre de la distance par rapport à leurs propres repères en matière de culture. À cet égard, il convient, pour les professeurs d'EPS, d'être attentifs à l'incidence éventuelle de leur propre passé de sportifs chevronnés. Un engagement durable de compétiteur ou de pratiquant à un bon niveau de performance est susceptible d'avoir des répercussions sur l'image qu'ils se font de l'excellence corporelle (et, par contrecoup, sur la manière de concevoir les contenus à transmettre en EPS). Dans ce domaine, les travaux d'Erbani (1983) 
montrent que l'habitus sportif forgé lors d'une pratique compétitive régulière à un bon niveau a des effets notables sur les conceptions pédagogiques développées par des étudiants préparant le professorat d'EPS.

\section{La méthodologie}

T es matériaux empiriques sur lesquels s'appuient les analyses proviennent de L deux principales sources. La nature des activités physiques servant de support aux séquences d'enseignement en EPS ainsi que les modalités de pratique valorisées dans cette discipline sont appréhendées, en premier lieu, à partir des textes officiels les plus récents : programmes d'enseignement fournissant les grandes orientations, documents d'accompagnement permettant de cerner de manière précise les objectifs poursuivis, textes officiels régissant les épreuves d'EPS du baccalauréat.

Cette première source est complétée par les données de deux enquêtes nationales menées par les services statistiques du ministère de l'Éducation nationale (Direction de l'Évaluation, de la Prospective et de la Performance) au cours de l'année 2006. Dans le cadre d'une convention signée entre la DEPP et nos deux équipes de recherche, nous avons pu réaliser l'analyse secondaire de ces enquêtes. Centrées sur l'EPS, elles ont permis d'interroger par questionnaire, à partir d'un échantillonnage national, 1954 élèves et 1317 enseignants. La base de sondage choisie est constituée des collèges, des lycées généraux et professionnels, publics et privés de la métropole et des départements d’Outre-mer. La méthode d'échantillonnage utilisée est le sondage stratifié. Trois critères ont été retenus pour réaliser cette stratification : la taille des établissements, le fait qu'ils soient classés ou non en zone d'éducation prioritaire (ZEP), le fait qu'ils soient implantés en zone urbaine ou en zone rurale. Ainsi, 360 collèges, 192 lycées généraux et 177 lycées professionnels ont été tirés au sort. Dans chaque établissement, un ou plusieurs élèves et professeurs ont été, à leur tour, tirés au sort. Le nombre d'élèves et de professeurs tirés au sort varie de 1 à 3 en fonction de la taille de l'établissement. Les taux de réponse sont plutôt élevés : 89\% pour les élèves et $90,3 \%$ pour les enseignants (ils sont un peu plus élevés en collège qu'en lycée). La représentativité des répondants appréciée selon les trois critères de stratification a été jugée très satisfaisante (cf. le rapport de Benhaim-Grosse 2007).

Signalons que lors du transfert des données par la DEPP, un certain nombre de données (sociologiquement pertinentes) n'ont pas été communiquées : appartenance ou non des établissements à une ZEP, implantation de ceux-ci en zone urbaine ou zone rurale. Par ailleurs, aucune variable ne permet d'identifier précisément l'origine sociale des élèves. 


\section{Résultats}

T ne enquête réalisée en 1985 à l'échelon national révélait que, du point de point de vue de la répartition des activités physiques servant de support aux séquences d'enseignement en EPS, pour le second degré, trois disciplines étaient nettement sur-représentées : l'athlétisme, la gymnastique sportive et la natation ; d'autres étaient réduites à la portion congrue : activités physiques artistiques, activités physiques de pleine nature et sports de combat (Combaz 1991). Un peu plus de deux décennies plus tard, observe-t-on les mêmes tendances?

\section{Les activités pratiquées en EPS : une focalisation sur un noyau de pratiques jugées fondamentales?}

Les programmes d'enseignement de l'EPS les plus récents pour les lycées et les lycées professionnels insistent sur la nécessité de confronter les élèves à la diversité des expériences corporelles. Ainsi pour le lycée, "l'approche intégrée des classes de première et de terminale répond au souci de permettre à tous les élèves de faire des choix qui les engagent délibérément dans leur pratique tout en veillant à la diversité des activités physiques, sportives et artistiques programmées" (CNDP 2004b, 11).

Pour le lycée professionnel, il est indiqué que "le rôle de l'EPS est de former à long terme un citoyen lucide, autonome et créatif en favorisant l'accès au patrimoine culturel constitué de la diversité des activités physiques, sportives et artistiques" (MEN 2002, 2536).

En ce qui concerne le choix des activités physiques, les équipes enseignantes sont invitées à puiser au sein de deux grands regroupements : un ensemble commun permettant d'assurer une relative homogénéité nationale et un ensemble complémentaire autorisant la prise en compte de spécificités locales.

L'ensemble commun est constitué des activités suivantes : volley-ball, basket-ball, handball, football, rugby, badminton, tennis de table, courses, sauts, lancers, triathlon, natation, gymnastique artistique, gymnastique acrobatique, escalade, course d'orientation, danse, judo, lutte, course en durée.

L'ensemble complémentaire peut comprendre des activités telles que: la boxe française, les arts du cirque, les étirements, la gymnastique rythmique et sportive, la gymnastique aérobique, les techniques de relaxation, la musculation, le tir à l'arc, etc. En fonction des spécificités locales, chaque académie élabore annuellement une liste d'activités pouvant être pratiquées dans les établissements.

Incontestablement, les textes officiels ouvrent l'éventail des activités physiques envisageables dans le cadre des enseignements de l'EPS. Un certain nombre de formulations et d'orientations laissent cependant supposer que toutes les activités physiques n'ont pas nécessairement la même valeur. 
Prenons l'exemple des programmes pour les classes de seconde. Après avoir insisté sur la diversité des activités physiques proposées, les textes mentionnent que les élèves doivent être confrontés “à certaines de leurs pratiques sociales” et qu'ils doivent pouvoir "accéder à la signification culturelle de chacune" (CNDP 2004a, 12). On relève ici deux orientations assez claires : l'idée d'une sélection parmi tous les types d'expériences corporelles ("certaines de leurs pratiques sociales") ; le postulat selon lequel à chaque activité physique correspond une seule signification culturelle.

Par ailleurs, en présentant la liste des activités de l'ensemble commun, il est indiqué que ce sont "les activités les plus fréquemment pratiquées dans les établissements scolaires". Il est également précisé -et il faut le souligner- que "l'enseignement s'appuiera de façon prioritaire sur ces activités" (CNDP 2004a, 14). Ceci se traduit directement dans le choix proposé aux élèves : pour chaque niveau du cursus (seconde, première, terminale), au moins trois activités sont choisies dont deux issues de l'ensemble commun. Ce dernier est constitué principalement d'activités athlétiques, de sports collectifs et de sports de raquette (tennis de table et badminton). Les activités artistiques, les sports de combat et les activités physiques de pleine nature sont comparativement bien moins représentés.

Le curriculum formel -appréhendé ici à travers les programmes d'enseignement- fait cohabiter une logique d'ouverture de l'éventail des activités physiques envisageables et une logique de hiérarchie relative de celles-ci. Par rapport à ce qui est prescrit, que font les enseignants, notamment du point de vue de la répartition des activités physiques pratiquées?

L'analyse des données des deux enquêtes de la DEPP réalisées en 2006 permet de dresser le constat suivant : l'athlétisme occupe encore une place prédominante mais on note la montée en puissance des sports collectifs et des sports de raquette (tennis de table et badminton) qui prennent la place jadis octroyée à la gymnastique sportive et à la natation (tableau 1). Le collège se distingue quelque peu du lycée et du lycée professionnel en accordant encore à la gymnastique sportive une place de choix.

L'importance accordée aux différentes activités physiques dans les enseignements d'EPS varie sensiblement. Cette hiérarchisation apparaît de manière assez discrète dans les orientations des textes officiels. Elle est fortement accentuée par les choix des enseignants.

Ces choix se justifient par l'existence de contraintes matérielles qui restreignent l'éventail des pratiques (installations sportives disponibles, éloignement des sites de pratique, etc.). Mais au-delà, il y a l'idée selon laquelle il existerait des activités physiques dites incontournables susceptibles de constituer les principaux fondements de l'enseignement de l'EPS. Une majorité des enseignants interrogés par la DEPP $(61,8 \%)$ est de cet avis. Lorsqu'on leur demande de citer précisément la nature de ces activités, un trio émerge nettement : la natation, $34,8 \%$, les sports collectifs, $25,8 \%$ et l'athlétisme, 23,3\%. 
Tableau I : Activités physiques pratiquées dans le cadre des cours d'EPS* au cours de l'année scolaire 2005 - 2006 (en pourcentages)

\begin{tabular}{|c|c|c|c|c|}
\hline Activités physiques & Collège & Lycée & Lycée professionnel & Ensemble \\
\hline Athlétisme & 68,4 & 48,8 & 48,3 & 55,5 \\
\hline Volley-ball & 46,3 & 47,3 & 44,6 & 46,1 \\
\hline Badminton & 38,5 & 50,5 & 47,1 & 45,2 \\
\hline Course d'endurance & 53,8 & 21,5 & 29,7 & 35,4 \\
\hline Tennis de table & 35,6 & 28,2 & 32,3 & 32,1 \\
\hline Basket-ball & 38,2 & 24,8 & 30,7 & $3 I, 3$ \\
\hline Handball & 42,7 & 20,1 & 20,7 & 28,2 \\
\hline Gymnastique sportive & 47,6 & 15,0 & 8,6 & 24,5 \\
\hline Football & 19,0 & 8,3 & 31,2 & 19,1 \\
\hline Acrosport & 23,1 & 22,5 & 10,0 & 18,8 \\
\hline Natation sportive & 13,6 & 13,7 & 9,3 & 12,3 \\
\hline Musculation & 4,5 & 6,9 & 17,6 & 9,4 \\
\hline Escalade & 8,0 & 8,8 & 8,0 & 8,3 \\
\hline Rugby & 11,2 & 3,8 & 3,3 & 6,2 \\
\hline Danse & 7,1 & 5,3 & 4,0 & 5,5 \\
\hline Course d'orientation & 6,7 & 4,8 & $4, I$ & 5,3 \\
\hline Boxe & 7,8 & 2,9 & 2,3 & 4,5 \\
\hline Lutte & 9,4 & $\mathrm{I}, \mathrm{I}$ & 2,0 & 4,3 \\
\hline Ultimate & 7,1 & 2,7 & 2,8 & 4,3 \\
\hline Base-ball & 5,5 & 3,0 & 2,7 & 3,8 \\
\hline Arts du cirque & 7,1 & 2,0 & $\mathrm{I}, 3$ & 3,6 \\
\hline Tennis & 3,0 & $\mathrm{I}, 7$ & 2,5 & 2,4 \\
\hline Gymnastique aérobique & 0,7 & 2,0 & 3,3 & 1,9 \\
\hline Hockey & 3,0 & 0,6 & 0,5 & $\mathrm{I}, 4$ \\
\hline Ski alpin & $\mathrm{I}, 3$ & $\mathrm{I}, 2$ & 1,3 & 1,3 \\
\hline Judo & 1,9 & $\mathrm{I}, \mathrm{I}$ & 0,5 & 1,2 \\
\hline
\end{tabular}

* Seules les activités pratiquées par plus de $\mathrm{I} \%$ des élèves ont été retenues (tous types d'établissement confondus)

Lecture : 1085 élèves sur les 1954 interrogés en 2006 (collèges, lycées et lycées professionnels confondus) ont pratiqué l'athlétisme dans le cadre des cours d'EPS, soit $55,5 \%$. 
Qu'est-ce qui peut expliquer la place prédominante accordée à ces activités ? La natation représente probablement un cas particulier car l'argument lié à la sécurité (apprendre à nager pour éviter de se noyer) est indiscutable. Ce type de justification ne tient pas pour les sports collectifs et l'athlétisme. Leur rayonnement international (compétitions à l'échelle mondiale, forte présence aux Jeux Olympiques, etc.), fait considérer ces deux familles d'activités par une majorité d'enseignants comme ayant une dimension universelle et doivent, à ce titre, être proposées, à la plupart des élèves.

Un autre type de justification consiste à penser qu'il existe des transferts d'apprentissage intervenant entre ces activités physiques à caractère universel et les autres considérées comme plus "secondaires". Les seuls travaux scientifiques consacrés à cette question réfutent une telle affirmation (Dugas \& Parlebas 1998).

Les interrogations formulées ci-dessus ne doivent pas être interprétées comme une position relativiste qui nierait l'existence de critères intrinsèques permettant de hiérarchiser les contenus culturels en vue de les transmettre dans le cadre scolaire. Nous questionnons simplement la validité des critères qui, en définitive, semblent être retenus pour justifier la hiérarchie des activités physiques sur laquelle l'enseignement de l'EPS prend appui.

Il y a tout lieu de penser que, dans l'imaginaire d'une majorité d'enseignants d'EPS, certaines activités physiques restent associées aux loisirs et à la détente et qu'elles ne peuvent, de ce fait, faire l'objet d'apprentissages sérieux, dignes de figurer dans les programmes scolaires. Ainsi, les activités physiques de pleine nature, les jeux traditionnels, la relaxation ou l'expression corporelle apparaissent comme des pratiques secondaires.

En prenant en considération la nature des activités physiques pratiquées par les élèves, la légitimité de la culture corporelle sur laquelle repose l'enseignement de l'EPS apparaît fondée sur un choix restreint de pratiques auxquelles -à tort ou à raison- un statut privilégié est accordé. Remarque-t-on le même type de tendance pour les modalités de pratique?

\section{Une préférence pour les formes de pratique reposant sur l'affrontement et la production de performances}

Une manière commode d'appréhender les modalités de pratique valorisées à l'école consiste, dans un premier temps, à rendre compte des objectifs officiellement assignés à l'enseignement de l'EPS.

Pour le lycée, les programmes mentionnent cinq principaux objectifs : réaliser une performance mesurée à une échéance donnée ; adapter ses déplacements à des environnements multiples, variés et nouveaux ; réaliser des actions à visée artistique ou esthétique ; conduire un affrontement individuel ou collectif ; orienter et développer les effets de l'activité physique en vue de l'entretien de soi 
(CNDP 2004b, 12). Pour chaque activité physique, les textes officiels précisent le type de compétences attendues, en liaison avec les cinq objectifs précités.

En ce qui concerne l'objectif "conduire un affrontement individuel ou collectif", incontestablement la logique compétitive prime. Le vocable utilisé est dénué de toute ambiguïté. Pour l'ensemble des sports collectifs et des sports de raquette, il s'agit de battre l'adversaire et de remporter la victoire. Quelques exemples suffisent à illustrer cette orientation. En handball, il s'agit de "rechercher le gain d'une rencontre par la mise en place d'une attaque fondée sur l'occupation permanente de l'espace de jeu (...)". Au tennis de table, il faut "proposer et mener à leur terme (...) des projets tactiques pour obtenir le gain de rencontres face à des adversaires identifiés et de niveau proche" (CNDP 2004b, 13-14).

Sans doute pour des raisons de sécurité, les orientations fournies pour les sports de combat échappent partiellement à cette logique du gain. En judo, il s'agit "en situation de randori, d'utiliser ses ressources pour affronter un partenaire ou un adversaire en élaborant et en mettant en œuvre un projet tactique" (CNDP 2004b, 15). Les termes ne sont pas anodins. Il s'agit, en premier lieu d'un randori, c'est-à-dire d'un combat généralement mené à l'entraînement. L'objectif n'est pas nécessairement de battre l'adversaire (comme en compétition) mais de le mettre en difficulté. Par ailleurs, le texte laisse ouverte la possibilité d'opter entre partenaire et adversaire. Ce qui laisse supposer une certaine distance prise avec la logique d'affrontement.

Sur l'ensemble des pratiques constituant l'ensemble commun, pour sept activités sur vingt (c'est-à-dire 35\%), la logique sportive compétitive prédomine.

Les orientations officielles sont aussi marquées par une dimension institutionnelle issue des fédérations sportives nationales ou internationales. Les activités physiques doivent être utilisées en EPS en respectant les caractéristiques et les règlements définis par ces fédérations pour les pratiques extrascolaires. C'est particulièrement net pour les activités gymniques. Ainsi, en gymnastique au sol, il faut "concevoir, construire et réaliser devant un public (...) un enchaînement (...) qui intègre les dimensions acrobatiques et esthétiques de l'activité gymnique et du code de référence" (CNDP 2004b, 15). L'évocation du code de référence illustre la dimension institutionnalisée de la pratique, celle qui renvoie aux règlements en usage lors des compétitions organisées par les instances nationales et internationales de gymnastique).

On retrouve cette orientation pour les activités athlétiques. Par exemple, pour le lancer de javelot ou le saut en hauteur, il convient de "se préparer et de réaliser la meilleure performance avec un nombre limité de tentatives" (CNDP 2004b, 14). Ce qui correspond pleinement au type de prestation attendu lors des rencontres sportives extrascolaires.

Le lien avec la pratique institutionnalisée (hors de l'école) est un peu plus lâche pour les activités artistiques et les activités physiques de pleine nature. En 
danse, il s'agit de "proposer un traitement chorégraphique qui questionne de façon personnelle et, dans les registres moteurs propres à la danse, une démarche de composition identifiée dans une œuvre”. Pour l'escalade, il s'agit, en position de premier de cordée, de "conduire son déplacement (...) selon des itinéraires variés afin de réaliser une performance tout en conservant assez de lucidité pour assurer sa sécurité" (CNDP 2004b, 15).

Comment ces différentes orientations sont-elles relayées (ou non) à l'échelon de l'établissement par les enseignants ?

Plusieurs recherches qualitatives récentes montrent que, dans la plupart des séquences observées, la compétition est présente même si elle se manifeste sous des formes et avec une intensité variables selon les situations : tournois, relais, décompte des points donnant lieu à un classement, etc. (Moreno 2006, Vigneron 2006). Pour justifier pédagogiquement la priorité donnée à cette modalité de pratique, il n'est pas rare que les enseignants évoquent le caractère ludique et motivant de cette approche.

Par ailleurs, une étude quantitative, centrée sur le collège, montre que le sport -défini au sens strict d'activité physique institutionnalisée dont la principale logique repose sur l'affrontement codifié (Parlebas 1999) - constitue, pour 43,6\% des professeurs interrogés, une référence prioritaire pour élaborer le contenu de leurs séquences d'enseignement (Poggi 2007).

Les enseignants d'EPS se montrent également très attachés à la dimension institutionnelle des activités physiques telle qu'elle apparaît notamment à travers une série de règlements édictés par les instances fédérales extrascolaires. Dans cette perspective, chaque activité est ainsi caractérisée par des spécificités -les professeurs parlent de logique interne- qu'il faut absolument respecter sous peine de dénaturer la pratique en question. Une grande majorité des enseignants interrogés par la DEPP semble partager cette position : 71,6\% d'entre eux estiment qu'il est essentiel de ne pas modifier sur le plan didactique les caractéristiques principales ('logique interne') des activités physiques pratiquées par les élèves en EPS.

Un autre élément de la pratique des activités physiques retient toute l'attention des professeurs d'EPS : produire une performance. Ce trait constitutif de la pratique sportive compétitive apparaît comme devant être pris en compte en EPS : $57 \%$ des enseignants interrogés par la DEPP sont plutôt d'accord ou tout à fait d'accord avec l'idée que le niveau de performance est le meilleur révélateur de l'efficacité du travail.

En définitive, la culture corporelle sur laquelle repose l'enseignement de l'EPS en France, dans le second degré, est caractérisée par deux aspects : la référence au sport compétitif et la dimension institutionnelle des activités physiques. À cet égard, on peut supposer que l'habitus sportif des professeurs d'EPS, leur passé de compétiteurs imprègnent en profondeur leurs conceptions des con- 
tenus enseignés. Les données de la DEPP montrent que leur engagement (présent et passé) dans la sphère sportive extrascolaire est relativement marqué (tableau 2).

\section{Tableau 2 : Pratiques, qualifications et investissements sportifs des professeurs d'EPS (en pourcentages)}

\begin{tabular}{|l|c|}
\hline Possède un diplôme fédéral d'entraîneur & 38,2 \\
\hline Possède un diplôme fédéral d'arbitre & 12,2 \\
\hline Possède un diplôme d'État d'éducateur sportif & 30,1 \\
\hline A eu une pratique sportive de haut niveau & 35,7 \\
\hline Est actuellement investi dans un club sportif & 58,1 \\
\hline Est investi dans un club sportif en tant que pratiquant sans faire de compétition & 22,4 \\
\hline Est investi dans un club sportif en tant que pratiquant en faisant de la compétition & 30,2 \\
\hline Est investi dans un club en tant qu'entraîneur & 23,7 \\
\hline Est investi dans un club en tant que dirigeant & 16,7 \\
\hline Est investi dans un club en tant qu'arbitre & 6,2 \\
\hline
\end{tabular}

La légitimité de ces choix en matière de pratiques corporelles peut-elle véritablement être mise à l'épreuve comme le laisse supposer le titre de cet article ? Sans être en mesure de la remettre fondamentalement en question pour l'instant, deux sources de tensions apparaissent cependant: les aspirations des élèves et l'évolution de la pratique des activités physiques hors de l'école.

\section{Des décalages avec les attentes des élèves et l'évolution de la pratique des activités physiques en dehors de l'école?}

Commençons par la nature des activités physiques. Plusieurs études antérieures ont montré qu'il existe un hiatus entre ce que propose l'école et les attentes des élèves (Secrétariat d'État auprès du ministre de la Qualité de la vie, Jeunesse et Sport 1976, Parlebas 1986, Combaz 1991). Qu'observe-t-on pour l'année scolaire 2005-2006?

Les résultats consignés dans le tableau 3 font apparaître un clivage assez net entre des activités très peu souvent pratiquées et celles qui le sont fréquemment. Pour les premières, les souhaits de pratique des élèves sont élevés ; pour les secondes, plutôt faibles. Les pratiques et les souhaits sont équilibrés pour une minorité d'activités : course d'endurance, course d'orientation et football. 
Tableau 3 : Activités physiques pratiquées en EPS
et souhaits de pratique des élèves (en pourcentage)

\begin{tabular}{|c|c|c|c|}
\hline Activités physiques & $\begin{array}{c}\text { Activités } \\
\text { pratiquées en EPS }\end{array}$ & $\begin{array}{l}\text { Pratique } \\
\text { souhaitée* }\end{array}$ & $\begin{array}{l}\text { Différences entre } \\
\text { pratiques et souhaits }\end{array}$ \\
\hline Plongée sous-marine & 0 & 36,5 & $-36,5$ \\
\hline Canoë-kayak & 0,5 & 33,5 & $-33,0$ \\
\hline Parapente & 0 & 29,7 & $-29,7$ \\
\hline VTT & 0,3 & 26,9 & $-26,6$ \\
\hline Ski alpin & $\mathrm{I}, 3$ & 27,1 & $-25,8$ \\
\hline Tennis & 2,4 & 28,1 & $-25,7$ \\
\hline Planche à voile & 0,1 & 25,3 & $-25,2$ \\
\hline Escalade (site naturel) & 7,2 & 32,2 & $-25,0$ \\
\hline Boxe & 4,5 & 26,4 & $-21,9$ \\
\hline Escrime & 0 & 20,7 & $-20,7$ \\
\hline Escalade (sur mur) & $\mathrm{I}, \mathrm{I}$ & 21,8 & $-20,7$ \\
\hline Voile & 0 & 19,2 & $-19,2$ \\
\hline Roller & 0,4 & 19,5 & $-19,1$ \\
\hline Hockey & $\mathrm{I}, 4$ & 20,4 & $-19,0$ \\
\hline Alpinisme & 0 & 18,7 & $-18,7$ \\
\hline Musculation & 9,4 & 26,4 & $-17,0$ \\
\hline Cyclisme & 0 & 16,2 & $-16,2$ \\
\hline Base-ball & 3,8 & 19,4 & $-15,6$ \\
\hline Ski nordique & 0 & 14,9 & $-14,9$ \\
\hline Judo & $\mathrm{I}, 2$ & 12,9 & $-11,7$ \\
\hline Rugby & 6,2 & 16,8 & $-10,6$ \\
\hline Natation synchronisée & 0,1 & 10,5 & $-10,4$ \\
\hline Gymnastique aérobique & 1,9 & $\mathrm{II}, 4$ & $-9,5$ \\
\hline Arts du cirque & 3,6 & 11,8 & $-8,2$ \\
\hline Danse & 5,5 & 13,6 & $-8,1$ \\
\hline Ultimate & 4,3 & 11,6 & $-7,3$ \\
\hline GRS** & 0,1 & 6,5 & $-6,4$ \\
\hline Lutte & 4,3 & 10,7 & $-6,4$ \\
\hline Natation sportive & 12,3 & 18,6 & $-6,3$ \\
\hline
\end{tabular}




\begin{tabular}{|l|c|c|c|}
\hline Activités physiques & $\begin{array}{c}\text { Activités } \\
\text { pratiquées en EPS }\end{array}$ & $\begin{array}{c}\text { Pratique } \\
\text { souhaitée* }\end{array}$ & $\begin{array}{c}\text { Différences entre } \\
\text { pratiques et souhaits }\end{array}$ \\
\hline Course d'endurance & 35,4 & 35,4 & 0 \\
\hline Course d'orientation & 5,3 & 5,3 & 0 \\
\hline Football & 19,1 & 18,4 & 0,7 \\
\hline Handball & 28,2 & 19,9 & 8,3 \\
\hline Acrosport & 18,8 & 8,7 & 10,1 \\
\hline Tennis de table & 32,1 & 18,6 & 13,5 \\
\hline Basket-ball & 31,3 & 16,9 & 14,4 \\
\hline Gymnastique sportive & 24,5 & 9,3 & 15,2 \\
\hline Badminton & 45,2 & 19,2 & 26,0 \\
\hline Volley-ball & 46,1 & 16,6 & 29,5 \\
\hline Athlétisme & 55,5 & 10,5 & 45,0 \\
\hline
\end{tabular}

* Pratique souhaitée à l'école ou en dehors de celle-ci.

**GRS : gymnastique rythmique et sportive.

Lecture : 242 élèves sur les 1954 interrogés en 2006 ont pratiqué la natation sportive dans le cadre des cours d'EPS, soit I2,3\% ; I8,6\% souhaitent pratiquer cette activité à l'école ou en dehors de celle-ci.

En tête des activités plébiscitées par les élèves figurent les activités physiques de pleine nature. Viennent ensuite : le tennis, certains sports de combat (dont la boxe), les activités dites d'entretien (musculation, gymnastique aérobique, etc.) et les activités physiques artistiques (danse, gymnastique rythmique et sportive, etc.).

Plusieurs interprétations sont envisageables. La première consiste à évoquer l'effet de saturation inhérent à la fréquence, en EPS, de certaines activités (athlétisme, badminton, volley-ball, etc.). Par contraste, d'autres activités auraient la faveur des élèves parce que, précisément, elles ne sont pas souvent proposées dans le cadre scolaire. C'est le cas des activités physiques de pleine nature. Précisons que, pour ces dernières, l'engouement des élèves ne s'est jamais démenti depuis les années 1970 (Hoibian 2003).

Les souhaits des élèves sont sans doute conditionnés, en partie, par des modes. Peut s'interpréter ainsi la place occupée actuellement par les activités physiques dites d'entretien ou les pratiques urbaines (le roller par exemple).

Les souhaits des élèves semblent assez bien refléter l'évolution récente de la pratique des activités physiques hors de l'école, telle qu'elle apparaît dans l'une des dernières enquêtes nationales (MJSVA \& al. 2005).

Une question essentielle reste posée : dans quelle mesure -et jusqu'oùl'école doit-elle tenir compte de ces évolutions? Elle se pose d'autant plus que 
les décalages évoqués à propos de la nature des activités physiques se manifestent également au sujet des modalités de pratique.

Lorsqu'on interroge les élèves sur leurs préférences en matière de pratiques physiques, deux principales tendances émergent. La première révèle une relative distance par rapport au modèle sportif compétitif : 52,4\% des élèves déclarent pratiquer les activités physiques pour le plaisir, sans se soucier de classement ni de performance ; $31,7 \%$ seulement d'entre eux mentionnent pratiquer parce que c'est une compétition qui permet de réaliser une performance et d'affirmer ses capacités.

Par ailleurs, une bonne proportion d'élèves $(55,9 \%)$ souhaite pratiquer les activités physiques en dehors de toute structure officielle (clubs sportifs, associations de loisirs, etc.) ; 36,2\% seulement préfèrent pratiquer en club. Cette tendance semble corroborer ce qu'on observe à plus grande échelle (tous âges confondus). L'enquête nationale la plus récente sur la pratique des activités physiques et sportives en France montre que les deux tiers pratiquent de manière autonome, c'est-à-dire en dehors de toute structure (MJSVA \& al. 2005).

En définitive, au sujet de la nature des activités physiques ou des modalités de pratique, il existe un décalage assez net entre ce que propose l'école -dans le cadre des enseignements de l'EPS-, les aspirations des élèves et l'évolution extrascolaire de la pratique des activités physiques.

\section{Conclusion}

F

ace à ce constat, il serait tentant d'adopter une position tranchée, comme l'a fait Vulliamy à propos de l'enseignement de la musique, pour dénoncer le caractère ethnocentrique de la culture corporelle valorisée par l'école. Il paraît plus fructueux d'aborder le décalage observé à travers la dialectique continuité/ rupture : l'EPS ne peut pas se couper totalement des évolutions sociales majeures, notamment en termes d'évolution des pratiques sportives extrascolaires (avec, par exemple, la progression spectaculaire des activités physiques de pleine nature, des pratiques urbaines ainsi que des activités physiques dites d'entretien) ; néanmoins, elle ne peut pas coller servilement à cette évolution sans en interroger les fondements et les apports originaux ; elle ne peut pas, non plus, tomber dans une forme de démagogie consistant à s'adapter naïvement aux attentes et à la culture première des élèves sans se soucier de les amener vers d'autres horizons culturels.

L'actualité institutionnelle offre l'opportunité d'aborder une question à peine esquissée ici : celle de la culture commune. L'arrêté du 5 juillet 2008 présentant les nouveaux programmes d'EPS pour le collège intègre pleinement cette dimension en précisant la contribution de la discipline aux acquisitions du socle commun. Il est explicitement mentionné que "par la pratique scolaire d'activités 
physiques sportives et artistiques, l'enseignement de l'EPS garantit à tous les élèves une culture commune" (MEN 2008, 1). Cette culture commune peut-elle être constituée d'un noyau restreint d'activités physiques dûment sélectionnées ou doit-elle prendre appui sur une large gamme de pratiques? L'architecture des programmes du collège comprend une liste nationale d'activités physiques très ouverte, calquée sur le modèle du lycée. Le texte insiste aussi (comme au lycée) sur la nécessité de faire vivre aux collégiens des expériences corporelles variées. Ce qui contraste quelque peu avec les résultats observés : actuellement, les activités physiques le plus souvent pratiquées sont: l'athlétisme, la gymnastique sportive (au sol et aux agrès), les sports collectifs et les sports de raquette (tableau 1). Le texte officiel n'indique nullement que ces activités-là constituent les principaux éléments d'une culture commune. Les enseignants d'EPS de collège (comme leurs collègues des lycées) vont-ils infléchir leurs choix ou reconduire les orientations actuelles? Il ne s'agit pas d'une querelle d'experts. Le choix des activités physiques et des modalités de pratique renvoie à la question des inégalités scolaires. Des travaux récents sur le lycée montrent que les contenus en vigueur mettent en difficulté les élèves de milieux modestes (Vigneron 2005) et, plus encore, les filles (Combaz \& Hoibian 2008). Ce qui pose la question du rôle que l'EPS joue dans la production des inégalités scolaires. Ceci n'a pas échappé au législateur puisque, dans le programme des collèges, il est stipulé que "l'EPS, à tous les niveaux de la scolarité, vise la réussite de tous les élèves" (MEN 2008, 1). Par ailleurs, le texte évoque la notion de "pratique scolaire". Ce terme suggère de prendre une certaine distance vis-à-vis des pratiques extrascolaires (sans être totalement en rupture avec celles-ci) : "l'apprentissage se fait au travers de pratiques scolaires issues de pratiques sociales, aménagées en fonction des impératifs éducatifs" (MEN 2008, 2). Cette orientation va dans le sens des propositions pédagogiques du Collectif d'étude disciplinaire et de réflexion sur l'enseignement (CEDRE), en particulier celle qui a pour ambition de construire une "culture scolaire des activités physiques en EPS" (Dhellemmes 2007). Dans cette perspective, les contenus d'enseignement sont élaborés en prenant une certaine distance avec les pratiques extrascolaires (notamment celles qui se pratiquent dans les structures institutionnalisées). En d'autres termes, les caractéristiques intrinsèques des activités physiques (telles qu'elles existent dans la sphère extrascolaire) peuvent faire l'objet de quelques aménagements en fonction de la spécificité de l'environnement scolaire et selon les objectifs propres de l'enseignant. Cela se traduit concrètement par une prise en compte des capacités (et des difficultés) des élèves. En volley-ball, par exemple, il est possible pour le joueur qui occupe le poste de passeur de bloquer momentanément la balle ; ce qui donne à ses partenaires le temps et l'occasion de construire une attaque convenable.

Il reste à vérifier qu'en procédant de la sorte, on parvienne réellement à construire une culture corporelle commune permettant à tous les élèves de s'épa- 
nouir. Il importe également d'analyser les effets d'une telle orientation sur les inégalités de réussite. On serait ainsi en mesure d'apprécier le rôle que pourrait jouer l'EPS dans le processus de démocratisation de l'enseignement.

\section{Références bibliographiques}

BENHAIM-GROSSE J. 2007 Image du sport scolaire et pratiques d'enseignement au collège et au lycée, Paris, DEPP

BOLTANSKI L. 1971 "Les usages sociaux du corps", Annales ESC-1, 205-233

CNDP (Centre National de Documentation Pédagogique) 2004a Programmes d'éducation physique et sportive. Classe de seconde générale et technologique, Paris, CNDP

CNDP 2004b Programmes d'éducation physique et sportive. Classe de première et de terminale, Paris, CNDP

COMBAZ G. \& HOIBIAN O. 2008 "Le rôle de l'école dans la construction des inégalités de sexe. L'exemple de l'éducation physique et sportive", Travail, genre et sociétés-20, $129-150$

COMBAZ G. 1991 "Quelles pratiques corporelles à l'école ? Curriculum prescrit, curriculum réel et attentes des élèves", Science et motricité-15, 23-29

DEMAILLY L. 1985 "Contribution à une sociologie des pratiques pédagogiques", Revue française de sociologie-XXVI-1, 96-119

DHELLEMMES R. 2007 "Pour une culture scolaire des APSA en EPS. Contribution du CEDRE”, Revue éducation physique et sport-328, 61-68

DUGAST E. \& PARLEBAS P. 1998 "Transfert d'apprentissage et domaines d'action motrice", Revue éducation physique et sport-270, 41-47

ERBANI E. 1983 "Influence de l'expérience sportive de compétition sur la pratique pédagogique des étudiants en EPS”, Motricité humaine-1, 3-11

FORQUIN J.-C. 1996 École et culture. Le point de vue des sociologues britanniques, Bruxelles, De Boeck

HOIBIAN O. 2003 Du plein air au cinquième domaine. Évolution du statut des activités physiques de pleine nature en EPS, in Terret T. dir. Éducation physique, sport et loisir, 1970-2000, Paris, éditions AFRAPS, 69-88

ISAMBERT-JAMATI V. \& GROSPIRON M.F. 1984 "Types de pédagogie du français et différenciation sociale des résultats. L'exemple du 'travail autonome' au deuxième cycle long", Études de linguistique appliquée-54, 69-97

MEN (Ministère de l'Éducation Nationale) 2002 "Programme d'enseignement de l'éducation physique et sportive pour les CAP, BEP et les baccalauréats professionnels", BOEN-39, 2536-2548

MEN (Ministère de l'Éducation Nationale) 2008 "Programme de l'enseignement d'éducation physique et sportive pour les classes de sixième, de cinquième, de quatrième et de troisième au collège", Bulletin officiel spécial-6, 1-9

MJSVA (Ministère de la Jeunesse, des Sports et de la Vie Associative) \& INSEE $2005 \mathrm{La}$ pratique des activités physiques et sportives en France, Paris, MJSVA, INSEP 
MORENO C. 2006 Formes de mixité en EPS et inégalités de réussite entre filles et garçons, in Cogérino G. dir. La mixité en éducation physique et sportive. Paroles, réussites, différenciations, Paris, éditions de la Revue EPS, 40-54

PARLEBAS P. 1986 Éléments de sociologie du sport, Paris, PUF

PARLEBAS P. 1999 Jeux, sports et sociétés. Lexique de praxéologie motrice, Paris, INSEP

POCIELLO C. 1981 La force, l'énergie, la grâce et les réflexes. Le jeu complexe des dispositions culturelles et sportives, in Pociello C. dir. Sports et société. Approches socioculturelles des pratiques, Paris, Vigot, 171-237

POGGI M.P. 2007 "Le modèle sportif : une stratégie de recours en établissement défavorisé", Science et motricité-61, 101-129

Programmes d'éducation physique et sportive. Classe de première et de terminale, 2004b Paris, CNDP

Programmes d'éducation physique et sportive. Classe de seconde générale et technologique, 2004a, Paris, CNDP

SECRÉTARIAT D'ÉTAT AUPRÈS DU MINISTRE DE LA QUALITÉ DE LA VIE, JEUNESSE ET SPORT 1976 Les lycéens et les collégiens face aux activités sportives, éducatives et culturelles, Paris, Secrétariat d'État auprès du ministre de la qualité de la vie, jeunesse et sport

TUPIN F. 1996 "Espace d'action des enseignants et démocratisation : l'exemple de l'enseignement de la narration écrite au collège", Revue française de pédagogie-115, 77-87

VIGNERON C. 2005 Les écarts de réussite en EPS aux examens entre filles et garçons, in Cogérino G. dir. Filles et garçons en EPS, Paris, éditions de la Revue EPS, 61-99

VIGNERON C. 2006 Sports collectifs : de l'ambition pour les filles, in Cogérino G. dir. La mixité en éducation physique et sportive. Paroles, réussites, différenciations, Paris, éditions de la Revue EPS, 55-89 